Check for updates

Cite this: RSC Adv., 2017, 7, 36444

Received 3rd June 2017

Accepted 6th July 2017

DOI: $10.1039 / c 7 r a 06203 f$

rsc.li/rsc-advances

\section{Molecular mechanism of helicase on graphene- based hybridization reaction platform for microRNA detection $\uparrow$}

\begin{abstract}
Xiaojun Fan, ${ }^{a}$ Yi Qi, ${ }^{a}$ Zhaoyi Shi, ${ }^{a}$ Yongkang Lv ${ }^{b}$ and Yujing Guo (D) *c
In this study, Escherichia coli RecQ helicase (RecQE) was introduced for the first time to demonstrate its molecular mechanism on a hybridization reaction for detecting microRNA (miRNA), using graphene oxide as a nano-quencher. In the miRNA detection system, the annealing of a "blocking" DNA strand to a fluorophore-labeled DNA probe (FDNA) effectively interfered with the non-specific binding of RecQE with FDNA. Aided by the motor force driven by adenosine triphosphate, the helicase-assisted hybridization system enhanced the hybridization of FDNA and miRNA, besides separating them. Compared with non-enzymatic detection system, the fluorescence signal of the RecQE-assisted system was increased by $17.17 \%$. More interestingly, RecQE did not affect stability of the formed FDNA-RNA duplex. This study will greatly benefit further studies of biological sensing processes based on nucleic acid hybridization in living cells, and has great enlightening significance for designing and improving miRNA detection methods by using recombinant helicases.
\end{abstract}

\section{Introduction}

Nowadays molecular biology studies are revolutionizing scientific diagnosis of human diseases. Molecular diagnostics help physicians better understand each patient's clinical conditions such as disease state, disease condition prediction, drug response, and treatment prognosis. ${ }^{1}$ The essence of molecular diagnostics lies in the use of biomarkers, including proteins, carbohydrates, or nucleic acids that are associated with pathological cells. ${ }^{2}$ As a group of endogenous noncoding RNAs, microRNAs (miRNAs, 18-25 nucleotides) play significant roles in regulating the expression of target messenger RNA (mRNA). ${ }^{3-6}$ Notably, frequent aberrant expression and functional implication of miRNAs in many human diseases have lifted these small cellular components to the ranks of biomarkers. ${ }^{7,8}$

Traditional methods for miRNA detection include northern blotting, ${ }^{9}$ RT-PCR ${ }^{10}$ microarrays ${ }^{11}$ and many others. Recently, various novel methods have been developed with a special focus on sensitivity and specificity, such as nanoparticle-derived probes, ${ }^{12-15}$ isothermal amplification, ${ }^{16-18}$ electrochemical methods, ${ }^{19-21}$ etc. Among different existing nanomaterials used

${ }^{a}$ College of Chemistry and Chemical Engineering, Taiyuan University of Technology, Taiyuan, 030024, China

${ }^{b}$ Key Laboratory of Coal Science and Technology, Ministry of Education and Shanxi Province, Taiyuan University of Technology, Taiyuan 030024, China

'Institute of Environmental Science, Shanxi University, Taiyuan 030006, China. E-mail: guoyj@sxu.edu.cn

$\dagger$ Electronic supplementary information (ESI) available. See DOI: $10.1039 / \mathrm{c} 7 \mathrm{ra06203f}$ for miRNA detection, graphene oxide (GO) can be functionalized as an ideal transducing element thanks to high water dispersibility, stable affinity toward hydrophobic and/or $\pi$ conjugated biomolecules, and the capability of fluorescence quenching. ${ }^{22-24}$ Former studies showed that GO could bind and quench dye-labeled single-stranded DNA (ssDNA) probes, while it had less binding affinity toward dsDNA or hybrids of DNA/ RNA. $^{25,26}$

Helicases are enzymes conserved from bacteria to humans that use ATP-driven motor force to unwind double-stranded DNA or RNA. ${ }^{27,28}$ Such molecular activity gives helicases great potential for assisting in certain molecular diagnostic systems, especially in hybridization-dependent biosensors. However, studies on coupling helicases with GO-based nucleic acid sensors are still lacking. ${ }^{29-32}$ From this standpoint, the effects of helicases on GO-based hybridization-dependent nucleic acid detection systems need deep on-going study to enucleate the enzyme-assisted detection mechanisms in vitro and to better understand the roles of these kinds of enzymes in intracellular miRNA detection.

Methodologically, varied affinities of ssDNA and dsDNA toward GO can be explored to study molecular mechanisms of helicases on a miRNA detection system. In this work, E. coli RecQ (RecQE) was selected as a model enzyme from the RecQ helicase family for our molecular mechanism study. In the platform, BDNA is annealed to FDNA to form a FDNA-BDNA duplex for the first step (Scheme 1A). This is followed by a step of closely absorbing such duplexes onto the GO surface to form a FDNA-BDNA-GO complex (Scheme 1B) with quenched fluorescence due to noncovalent $\pi-\pi$ stacking interactions between 


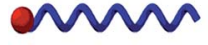

the hexagonal cells of graphene and the nucleobase ring structures. In the final step, RecQE and ATP are introduced to the system to form a FDNA-RNA duplex through structure switching (Scheme 1C), enabling augmentation of the fluorescent signal.

\section{Results and discussion}

\subsection{Investigating the function of a BDNA in helicase-assisted miRNA detection system}

In order to obtain higher detection sensitivity, the first problem to be solved is to minimize influence of background fluorescence due to the introduction of helicase into the miRNA detection system. Using BDNA was one of many attempts that can reduce the nonspecific binding of single strand FDNA with RecQE, which was expressed in E. coli cells and purified with a nickel sepharose column (Fig. S1†), to thereby improve stability of the detection system. Before exploring the function of BDNA, the quenching ability of GO was evaluated using fluorescence spectra of FDNA-BDNA in the presence of GO. As shown in Fig. S2, $\dagger$ addition of $6 \mu \mathrm{g} \mathrm{mL}{ }^{-1}$ GO resulted in complete fluorescence quenching of a $50 \mathrm{nM}$ FDNA-BDNA composite probe.

To investigate the fluorescence signal attenuation effect of BDNA on the RecQE-assisted system, a series of tests were conducted at different RecQE concentrations for both FDNAGO and FDNA-BDNA-GO systems without target miRNA. As shown in Fig. 1A, curves $a$ and $b$ had obviously different trends in fluorescence signal enhancement with increased RecQE concentration. This phenomenon indicated that FDNA-GO system interference was easier with RecQE than the FDNABDNA-GO system, and the formation of FDNA-BDNA duplex could effectively reduce the nonspecific binding of RecQE to FDNA. Besides, control tests using bovine serum albumin (BSA) obtained from serum in the FDNA-BDNA-GO system produced the expected low fluorescence signal at all concentrations (Fig. 1A-c), indicating that random proteins had much lower
A

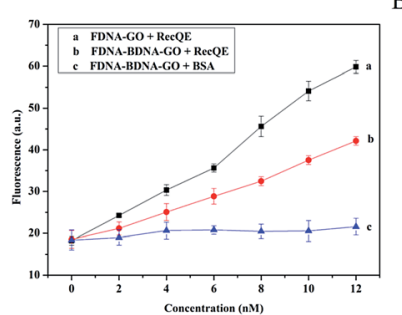

B

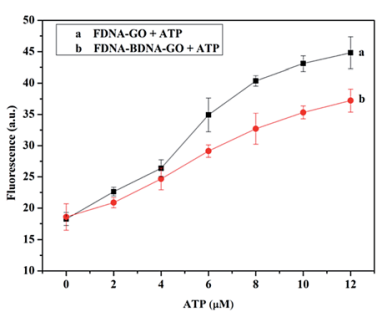

Fig. 1 Effect of BDNA on stability of the detection system. (A) (a) The effect of increasing RecQE concentration on the FDNA-GO system, (b) the effect of increasing RecQE concentration on the FDNABDNA-GO system, and (c) the effect of increasing BSA concentration on the FDNA-BDNA-GO system; (B) (a) the effect of increasing ATP concentration on the FDNA-GO system, (b) the effect of increasing ATP concentration on the FDNA-BDNA-GO system. RecQE concentrations: $2,4,6,8,10$, and 12 nM, ATP concentrations: 2, 4, 6, 8, 10 , and $12 \mu \mathrm{M}$.

nonspecific action with FDNA than RecQE. A small quantity of nonspecific binding of RecQE to the single stranded probe FDNA may result in dissociation of FDNA from the GO surface and fluorescence signal enhancement. The effects of ATP on both FDNA-GO and FDNA-BDNA-GO systems were similarly studied, and according to the results (Fig. 1B), the FDNABDNA-GO system showed a relatively better signal attenuation effect. Fluorescence resonance energy transfer (FRET) between GO and a fluorescence probe could be affected by ATP, and thereby enhance the fluorescence signal.

These results implied that the FDNA-BDNA-GO system was minimally interfered with by RecQE and other proteins.

Introducing BDNA to the miRNA detection system can accelerate formation of the FDNA-RNA duplex, ${ }^{33}$ and increase fluorescent signal generation upon addition of target miRNA. As shown in Fig. S3, $\dagger$ BDNA could greatly increase the fluorescent signal when the concentration of target miRNA was $25 \mathrm{nM}$, while fluorescent signal generation did not increase much when the target miRNA concentration was equal to FDNA (50 nM).

\subsection{Functional exploration and concentration optimization of RecQE and ATP in the FDNA-BDNA-GO system}

Although the FDNA-BDNA-GO system had certain stability, the fluorescence signal was enhanced to some extent with increasing RecQE concentration in the absence of target miRNA (Fig. 1A-b). Under such circumstances, the functions of helicase in the miRNA detection system need further studies. In this part, the hybridization reactions between FDNA-BDNA and target let-7a miRNA were tested in the presence of both RecQE and ATP inside the detection system. When the RecQE concentration was above $3 \mathrm{nM}$, signal enhancement tended to reach a stable level in the absence of ATP (Fig. 2A-a) which indicated that RecQE can anneal two complementary singlestranded nucleic acids without ATP. ${ }^{27}$ Compared with the hybridization test results with only RecQE added, fluorescence signal enhancement achieved by also adding ATP $(10 \mu \mathrm{M})$ - the source of driving motor force - showed an overall rise (Fig. 2Ab). Results showed that the fluorescence signal of the detection 
A

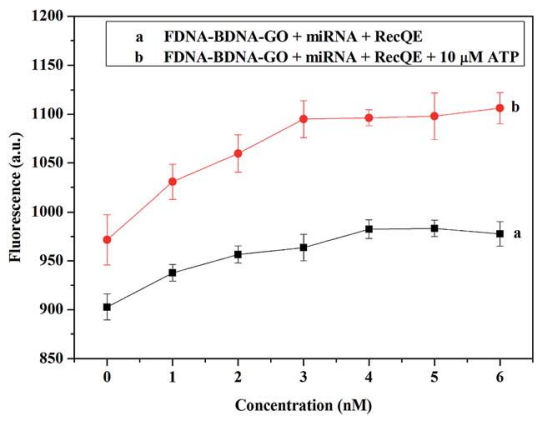

B

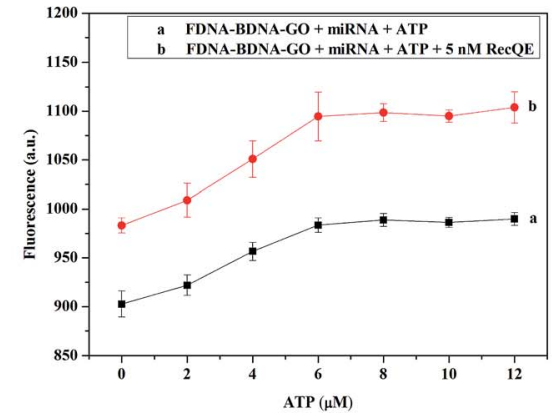

C

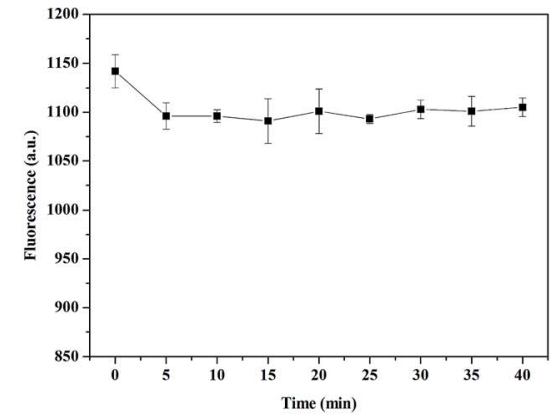

Fig. 2 Effects of RecQE and ATP on signal enhancement of the FDNA-BDNA-GO system. (A) The effect of increasing concentrations (0, 1, 2, 3, 4, 5, $6 \mathrm{nM})$ of RecQE without/with fixed ATP $(10 \mu \mathrm{M})$; (B) the effect of increasing ATP concentrations $(0,2,4,6,8,10,12 \mu \mathrm{M})$ without/with fixed RecQE (5 nM); and (C) the time effect of RecQE on the formation of FDNA-RNA duplex assembly. MiRNA concentration: $50 \mathrm{nM}$.

system assisted by RecQE using ATP displayed a remarkable enhancement (other than attenuation) of $17.17 \%$ over an enzyme-free reaction system. In some cases, as the helicaseinduced unwinding of incomplete dsDNA proceeded, the fluorescence signal decreased to some point because of the strong interaction of GO with unwound ssDNA. ${ }^{34}$ To discover the time effect of RecQE on formation of the FDNA-RNA duplex assembly, tests were deliberately designed and run to confirm the results above. As shown in Fig. 2C, fluorescence intensity changed very little from 0 to $40 \mathrm{~min}$. This result further proved that RecQE could not unwind the complete FDNA-RNA duplex structure. This motivating result may be attributed to the structure of closed dsDNA without ssDNA binding sites for helicase translocation. ${ }^{35,36}$

\subsection{Detection capability of let-7a miRNA}

After optimizing the RecQE-assisted FDNA-BDNA-GO system, the application of such assay was investigated for various

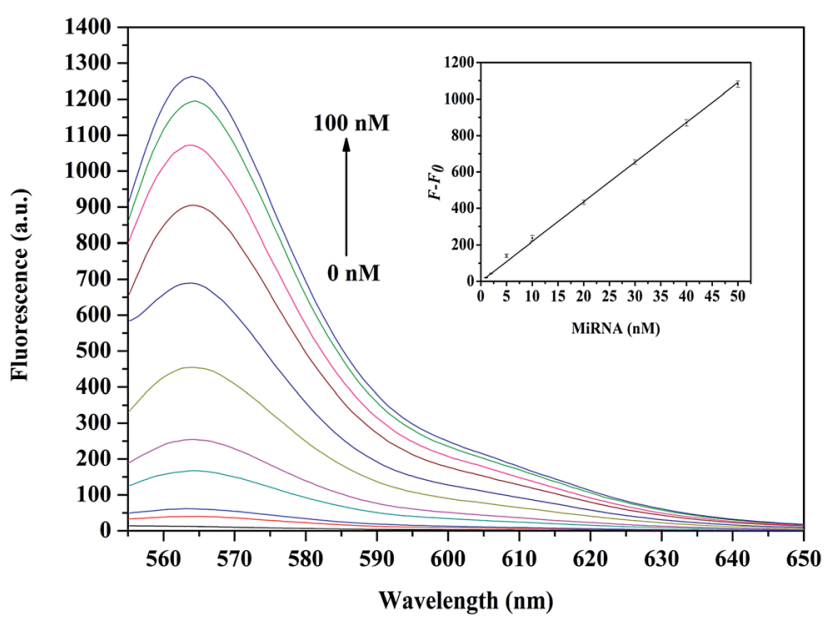

Fig. 3 Fluorescence spectra of the mixture solutions with different concentrations of let- $7 \mathrm{a}(0,1,2,5,10,20,30,40,50,80$, and $100 \mathrm{nM})$. Inset: curve fitting result for the relative fluorescence signal intensity versus corresponding target miRNA concentrations. Error bars were estimated from three repeating measurements. concentrations of let-7a miRNA in order to assess detection capability. As shown in Fig. 3, when the concentration of let-7a increased from 0 to $100 \mathrm{nM}$, a gradual increase in fluorescence intensity was observed, indicating dependence of fluorescence intensity upon the concentration of let-7a. By plotting the logarithmic value of let-7a concentration $v s$. fluorescence intensity, a fitting curve with a good correlation coefficient of 0.9985 could be obtained in the concentration range from $1 \mathrm{nM}$ to $50 \mathrm{nM}$ (Fig. 3, inset), and the detection limit of the method was calculated to be $0.18 \mathrm{nM}$ for miRNA based on the $3 \sigma$ rule. However, the fluorescence signal intensity did not show a linear relationship with let-7a concentrations in the range of 50$100 \mathrm{nM}$. The main reason might be that, when the concentration of let-7a was up to $50 \mathrm{nM}$, FDNA with fixed concentrations (say, $50 \mathrm{nM}$ ) was exhausted theoretically, resulting in little further increase of the fluorescence signal.

There are studies on target detection sensitivity based on equivalent binding models, i.e., one target molecule can only hybridize with one fluorescent DNA probe and thereby recovers one fluorophore from the GO surface. Compared with graphene oxide-based single-layer $\mathrm{MoS}_{2}$ nanoprobes and quantum dots models ${ }^{24,29}$ where detection limits for nucleic acids were 500 pM and $12 \mathrm{nM}$, a more sensitive detection limit was obtained in this helicase-assisted detection system. These results further suggested that RecQE had scarcely any effect on stability of the formed FDNA-RNA duplex assembly, and the proposed assay approach is appropriate for sensitive quantification of nucleic acids.

\subsection{Detection specificity of target let-7a miRNA}

Specificity of the proposed assay strategy was investigated by detecting let-7a, let-7f, let-7g, let-7i, and non-complementary RNA (NC) with the same concentration (50 nM) and blank control without any miRNA in reaction solutions. Fig. 4 displays fluorescence emission spectra of the RecQE-assisted FDNABDNA-GO system in the presence of different miRNAs. Fluorescence enhancements by the single-base-mismatched miRNA let-7f, two-base-mismatched miRNA let-7g, and four-basemismatched miRNA let-7i were $83.23 \%, 63.96 \%$, and $48.95 \%$ of that by the target let-7a, respectively. These results reveal 


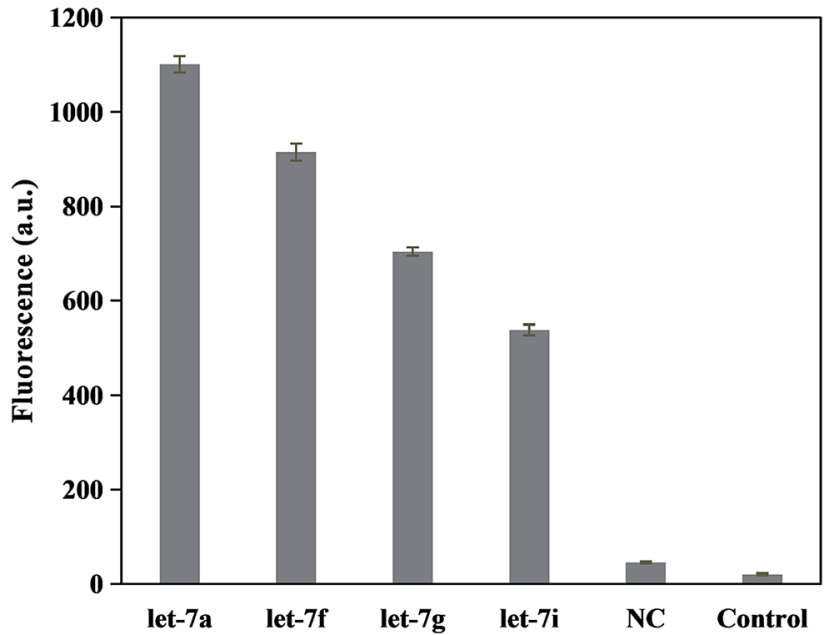

Fig. 4 Comparison of the fluorescence intensities produced by let-7a, let-7f, let-7g, let-7i, and NC. Control tests were run without any miRNA.

capability of the RecQE-assisted FDNA-BDNA-GO system to detect various base-mismatched miRNAs. Moreover, this study showed that non-complementary RNA and control tests had little effect on fluorescence signal enhancement of the FDNABDNA-GO system, indicating high specificity of the detection system.

\subsection{Kinetics and thermodynamics}

Kinetic behavior of the FDNA-BDNA-GO system assisted by RecQE and ATP was studied by monitoring fluorescence intensity as a function of time. Adsorption of the FDNA-BDNA duplex onto the GO surface was very fast at $37^{\circ} \mathrm{C}$. As shown in Fig. 5A-a, the fluorescence was effectively quenched by GO in even a shorter time than $5 \mathrm{~min}$. However, the process of forming double-stranded FDNA-RNA and releasing it from GO was relatively slow (Fig. 5A-b), taking about $30 \mathrm{~min}$ to complete the reaction.

Melting-temperature measurements were conducted to study the thermostability of FDNA-BDNA-GO and double-
A

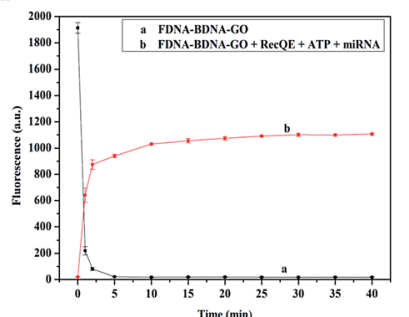

B

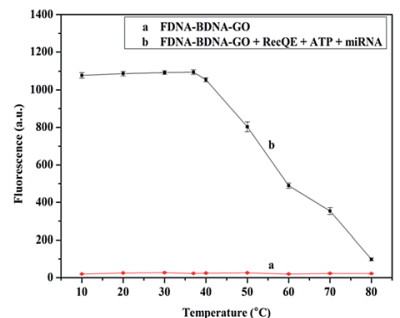

Fig. 5 Kinetic and thermodynamic characterizations of the RecQEassisted detection system. (A) (a) Fluorescence quenching of FDNABDNA by $G O$ as a function of time, (b) fluorescence restoration of the FDNA-BDNA-GO system by let-7a as a function of time; (B) (a) temperature effects on the fluorescence emission intensities of FDNA-BDNA-GO system, (b) temperature effects on the fluorescence signal of the detection system. stranded FDNA-RNA. The FDNA-BDNA-GO system was exposed to different temperatures between $10{ }^{\circ} \mathrm{C}$ and $80^{\circ} \mathrm{C}$ with $10{ }^{\circ} \mathrm{C}$ increments, allowing $30 \mathrm{~min}$ reaction and fluorescence signal monitoring. The FDNA-BDNA-GO complex displayed an excellent thermostability even at high temperatures, paralleling minimal change in fluorescence intensity at all the temperatures (Fig. 5B-a). However, when temperatures were above $37^{\circ} \mathrm{C}$, the formed FDNA-RNA complex started melting, resulting in a diminished fluorescence signal (Fig. 5B-b). It is worth mentioning that the highest fluorescence signal intensity of the RecQE-assisted detection system was detected at $37^{\circ} \mathrm{C}$.

\section{Conclusions}

For the first time, this study reports the molecular mechanism of helicase on a GO-based nucleic acid sensor with equivalent hybridization models in vitro. Comprehensive results showed that helicases had an important role in the detection process of a GO-based nucleic acid sensor, and the enzyme did not affect stability of the formed double-stranded FDNA-RNA duplex. In addition, BDNA can weaken the nonspecific interaction between RecQE and FDNA. Since helicases have an extensive applicability with hybridization reactions of nucleic acids, this work will benefit further studies of biological sensing processes based on nucleic acid hybridization.

\section{Experimental section}

\subsection{Materials and apparatus}

HPLC-purified DNA and miRNAs were synthesized and purified by Sangon Biotech Co., Ltd. (Shanghai, China). Sequences of the oligonucleotide probes and miRNAs are listed in Table 1 . Diethylpyrocarbonate (DEPC)-treated deionized water and ribonuclease inhibitor were purchased from Solarbio Biotechnology Co., Ltd. (Beijing, China). GO was synthesized from natural graphite according to the classic protocol with a modification of Hummers' method. ${ }^{37,38}$ pET24a-RecQE recombinant plasmid was constructed and presented by Hua Ren (Department of Biomedical Sciences, School of Life Science, East China Normal University, Shanghai 200241, China). Unless otherwise noted, all other chemicals were purchased from Sangon Biotech Co., Ltd. (Shanghai, China). DEPC-treated deionized water was used in all experiments.

Table 1 Sequences of the probes and microRNAs ${ }^{a}$

\begin{tabular}{ll}
\hline Name & Sequences $\left(5^{\prime}-3^{\prime}\right)$ \\
\hline FDNA & Cy3-AACTATACAACCTACTACCTCA \\
BDNA & TGAGGTA \\
Let-7a & UGAGGUAGUAGGUUGUAUAGUU \\
Let-7f & UGAGGUAGUAGAUUGUAUAGUU \\
Let-7g & UGAGGUAGUAGUUUGUACAGUU \\
Let-7i & UGAGGUAGUAGUUUGUGCUGUU \\
Non-complementary RNA & UUGUACUACACAAAAGUACUGG
\end{tabular}

${ }^{a}$ Note: different bases in let-7 were underlined. 
SDS-polyacrylamide gel electrophoresis (SDS-PAGE) was performed using a DYY-10C Electrophoresis Apparatus (Liuyi, Beijing, China). Ultrapure water was purified by a Simplicity UV Water Purification System (Millipore SAS, France). Fluorescence spectra were obtained using a Hitachi F-2700 Fluorescence Spectrophotometer (Hitachi, Japan).

\subsection{FDNA-BDNA-GO system}

The FDNA-BDNA-GO system contains $50 \mathrm{nM}$ FDNA and $50 \mathrm{nM}$ BDNA. Prior to reaction, FDNA and BDNA were both incubated in $1 \times$ SPSC buffer $\left(0.75 \mathrm{M} \mathrm{NaCl}\right.$ and $\left.50 \mathrm{mM} \mathrm{Na}_{2} \mathrm{HPO}_{4}, \mathrm{pH} 7.4\right)$ at $37^{\circ} \mathrm{C}$ to fold the FDNA-BDNA duplex assembly. After that, GO $\left(6 \mu \mathrm{g} \mathrm{mL} \mathrm{m}^{-1}\right)$ was added to the solution and incubated for $30 \mathrm{~min}$ at $37^{\circ} \mathrm{C}$.

\subsection{Optimization of RecQE and ATP on FDNA-BDNA-GO system}

Optimization of RecQE concentration in the FDNA-BDNA-GO system was performed in $1 \mathrm{~mL}$ of a FDNA-BDNA-GO system containing $50 \mathrm{nM}$ let-7a, $10 \mu \mathrm{M}$ ATP, $40 \mathrm{U}$ RNase inhibitor, and different concentration levels of $\operatorname{RecQE}(1,2,3,4,5$, and $6 \mathrm{nM})$. The final mixture was incubated at $37^{\circ} \mathrm{C}$ for $30 \mathrm{~min}$.

For optimizing ATP concentration in the FDNA-BDNA-GO system, the reaction was performed in $1 \mathrm{~mL}$ of a FDNA-BDNAGO system containing $50 \mathrm{nM}$ let-7a, $5 \mathrm{nM}$ RecQE, 40U RNase inhibitor, and different concentrations of ATP $(2,4,6,8,10$, and $12 \mu \mathrm{M}$ ). The final mixture was incubated at $37{ }^{\circ} \mathrm{C}$ for $30 \mathrm{~min}$.

For the time effect of RecQE on detection of the FDNA-RNA duplex assembly, FDNA (50 nM) and let-7a (50 nM) were first incubated together at $37^{\circ} \mathrm{C}$ for $1 \mathrm{~h}$ to perfectly fold into a FDNARNA duplex assembly, and then, $\mathrm{GO}\left(6 \mu \mathrm{g} \mathrm{mL} \mathrm{m}^{-1}\right)$ was added to the system and incubated for $30 \mathrm{~min}$ at $37^{\circ} \mathrm{C}$. Subsequently, RecQE $(5 \mathrm{nM})$ and ATP $(10 \mu \mathrm{M})$ were mixed with the solution, and the fluorescence signal was measured every five minutes $(0-$ $40 \mathrm{~min})$.

\subsection{Target miRNA detection}

The hybridization reaction was performed in $1 \mathrm{~mL}$ of the FDNABDNA-GO system containing $5 \mathrm{nM}$ RecQE, $10 \mu \mathrm{M}$ ATP, 40U RNase inhibitor, and let-7a in different concentrations. Subsequently, the mixture was incubated at $37^{\circ} \mathrm{C}$ for $30 \mathrm{~min}$.

\subsection{Fluorescence spectra}

Fluorescence of a Cy3-labeled DNA probe was excited at $540 \mathrm{~nm}$, and the resulting spectra were recorded between $555 \mathrm{~nm}$ and $650 \mathrm{~nm}$. The fluorescence emission maximized at $564 \mathrm{~nm}$. Spectrometer slits were set for $5 \mathrm{~nm}$ band pass and operating voltage at $700 \mathrm{~V}$.

\section{Conflicts of interest}

There are no conflicts of interest to declare.

\section{Acknowledgements}

This work was supported the Natural Science Foundation of Shanxi Province (No. 201603D321094) and the Young "Sanjin" Scholar Foundation of Shanxi Province, 2016. The authors thank Lihui Bai, Min Li and Lu Li for their help with some of the experiments.

\section{References}

1 J. Hoggatt, Mol. Diagn. Ther., 2011, 15, 53-55.

2 A. B. Chinen, C. M. Guan, J. R. Ferrer, S. N. Barnaby, T. J. Merkel and C. A. Mirkin, Chem. Rev., 2015, 115, 10530.

3 L. He, J. M. Thomson, M. T. Hemann, E. Hernando-Monge, D. $\mathrm{Mu}, \mathrm{S}$. Goodson, S. Powers, C. Cordon-Cardo, S. W. Lowe, G. J. Hannon and S. M. Hammond, Nature, 2005, 435, 828.

4 S. M. Johnson, H. Grosshans, J. Shingara, M. Byrom, R. Jarvis, A. Cheng, E. Labourier, K. L. Reinert, D. Brown and F. J. Slack, Cell, 2005, 120, 635-647.

5 J. J. Rossi, Cell, 2009, 137, 990-992.

6 A. Ventura and T. Jacks, Cell, 2009, 136, 586-591.

7 N. Chiorazzi, K. R. Rai and M. Ferrarini, N. Engl. J. Med., 2005, 352, 804-815.

8 A. Esquelakerscher and F. J. Slack, Nat. Rev. Cancer, 2006, 6, 259-269.

9 A. Válóczi, C. Hornyik, N. Varga, J. Burgyán, S. Kauppinen and Z. Havelda, Nucleic Acids Res., 2004, 32, e175.

10 C. Chen, D. A. Ridzon, A. J. Broomer, Z. Zhou, D. H. Lee, J. T. Nguyen, M. Barbisin, N. L. Xu, V. R. Mahuvakar, M. R. Andersen, K. Q. Lao, K. J. Livak and K. J. Guegler, Nucleic Acids Res., 2005, 33, e179.

11 W. Li and K. Ruan, Anal. Bioanal. Chem., 2009, 394, 11171124.

12 F. Degliangeli, P. Kshirsagar, V. Brunetti, P. P. Pompa and R. Fiammengo, J. Am. Chem. Soc., 2014, 136, 2264-2267.

13 D. W. Hwang, I. C. Song, D. S. Lee and S. Kim, Small, 2010, 6, 81-88.

14 Z. Lu, L. Zhang, Y. Deng, S. Li and N. He, Nanoscale, 2012, 4, 5840-5842.

15 Y. P. Zeng, G. Zhu, X. Y. Yang, J. Cao, Z. L. Jing and C. Y. Zhang, Chem. Commun., 2014, 50, 7160-7162.

16 H. Dong, J. Zhang, H. Ju, H. Lu, S. Wang, S. Jin, K. Hao, H. Du and X. Zhang, Anal. Chem., 2012, 84, 4587-4593.

17 W. Li, T. Hou, M. Wu and F. Li, Talanta, 2016, 148, 116-121.

18 H. Liu, L. Li, L. Duan, X. Wang, Y. Xie, L. Tong, Q. Wang and B. Tang, Anal. Chem., 2013, 85, 7941-7947.

19 A. Erdem and G. Congur, Talanta, 2014, 118, 7-13.

20 S. P. Jonstrup, J. Koch and J. Kjems, RNA, 2006, 12, 17471752.

21 F. Li, J. Peng, J. Wang, H. Tang, L. Tan, Q. Xie and S. Yao, Biosens. Bioelectron., 2014, 54, 158-164.

22 J. N. Lekitima, K. I. Ozoemena, C. J. Jafta, N. Kobayashi, Y. Song, D. Tong, S. Chen and M. Oyama, J. Mater. Chem. A, 2013, 1, 2821-2826. 
23 Z. Wang, J. Zhang, P. Chen, X. Zhou, Y. Yang, S. Wu, L. Niu, Y. Han, L. Wang, P. Chen, F. Boey, Q. Zhang, B. Liedberg and H. Zhang, Biosens. Bioelectron., 2011, 26, 3881-3886.

24 C. Zhu, Z. Zeng, H. Li, F. Li, C. Fan and H. Zhang, J. Am. Chem. Soc., 2013, 135, 5998-6001.

25 S. He, B. Song, D. Li, C. Zhu, W. Qi, Y. Wen, L. Wang, S. Song, H. Fang and C. Fan, Adv. Funct. Mater., 2010, 20, 453-459.

26 W. Qiang, W. Li, X. Li, X. Chen and D. Xu, Chem. Sci., 2014, 5, 3018-3024.

27 Y. Wu, J. Nucleic Acids, 2012, 2012, 140601.

28 D. L. Croteau, V. Popuri, P. L. Opresko and V. A. Bohr, Annu. Rev. Biochem., 2014, 83, 519-552.

29 H. Dong, W. Gao, F. Yan, H. Ji and H. Ju, Anal. Chem., 2010, 82, 5511-5517.

30 C. H. Lu, H. H. Yang, C. L. Zhu, X. Chen and G. N. Chen, Angew. Chem., Int. Ed., 2009, 121, 4879-4881.
31 L. Li, J. Feng, H. Liu, Q. Li, L. Tong and B. Tang, Chem. Sci., 2016, 7, 1940-1945.

32 L. Yang, C. Liu, W. Ren and Z. Li, ACS Appl. Mater. Interfaces, 2012, 4, 6450-6453.

33 J. Song, P. S. Lau, M. Liu, S. Shuang, C. Dong and Y. Li, ACS Appl. Mater. Interfaces, 2014, 6, 21806-21812.

34 H. Jang, Y. K. Kim, H. M. Kwon, W. S. Yeo, D. E. Kim and D. H. Min, Angew. Chem., Int. Ed., 2010, 122, 5839-5843.

35 S. X. Dou, P. Y. Wang, H. Q. Xu and X. G. Xi, J. Biol. Chem., 2004, 279, 6354-6363.

36 H. Q. Xu, E. Deprez, A. H. Zhang, P. Tauc, M. M. Ladjimi, J. C. Brochon, C. Auclair and X. G. Xi, J. Biol. Chem., 2003, 278, 34925-34933.

37 W. S. Hummers Jr and R. E. Offeman, J. Am. Chem. Soc., 1958, 80, 1339.

38 Y. Guo, S. Guo, J. Ren, Y. Zhai, S. Dong and E. Wang, ACS Nano, 2010, 4, 4001-4010. 